\title{
Salud mental y estrategias de aculturación en inmigrantes colombianos y peruanos en el norte de Chile
}

\author{
Alfonso Urzúa M. ${ }^{* 1}$, Susana Boudon Torrealba ${ }^{1}$ y Alejandra Caqueo-Urízar². \\ ${ }^{1}$ Escuela de Psicología, Universidad Católica del Norte. Antofagasta, Chile. \\ ${ }^{2}$ Departamento de Psicología, Universidad de Tarapacá, Arica, Chile.
}

Recibido, mayo 28/2015

Concepto de evaluación, julio 5/2016

Aceptado, agosto 4/2016
Referencia: Urzúa M., A., Boudon Torrealba, S. \& Caqueo-Urízar, A. (2017). Salud Mental y Estrategias de Aculturación en inmigrantes colombianos y peruanos en el Norte de Chile. Acta Colombiana de Psicología, 20(1), 70-79. DOI: 10.14718/ACP.2017.20.1.5

Resumen

\begin{abstract}
Mediante un diseño no experimental de tipo transversal se contrastó la hipótesis de que el estado de salud mental de los inmigrantes evaluados difiere según la estrategia de aculturación que utilicen. Para esto se evaluó la diferencia de medias en sintomatología, rol social y relaciones interpersonales entre los participantes agrupados por tipo de estrategia de aculturación (integración o biculturalidad, separación, aislamiento y asimilación) en una muestra de 431 inmigrantes peruanos y colombianos residentes en la ciudad de Antofagasta, Chile. Para evaluar la aculturación se utilizó la Escala revisada de actitudes hacia la aculturación de Basabe, Zlovina y Páez, y para evaluar la Salud Mental se aplicó el cuestionario de Autoevaluación OQ-45.2. Como resultado se encontró que la estrategia más utilizada por los colombianos fue la separación, y por los peruanos, la asimilación, además de que los primeros reportaron mayor presencia de síntomas de problemas de salud mental en todas las dimensiones y en la escala total, en comparación con la población peruana. En la muestra total, los individuos que utilizaron la estrategia asimilación presentaron un mayor reporte de sintomatología y dificultades en las relaciones interpersonales. Los datos aportan evidencia con respecto a que las estrategias de aculturación utilizadas incidirían en una mayor o menor presencia de sintomatología psicopatológica.
\end{abstract}

Palabras clave: salud mental, aculturación, inmigración.

\section{Mental bealth and psychological acculturation strategies in Peruvian and Colombian immigrants in northern Chile}

\begin{abstract}
Using a cross-sectional, non-experimental design, the hypothesis that the mental health state of assessed immigrants differs according to the acculturation strategy used, was tested. To this end, the mean difference in physiological symptoms, social roles and relationships among participants categorized by type of acculturation strategy, i.e. bicultural integration, separation, isolation and assimilation, was evaluated. The sample consisted of 431 Peruvian and Colombian immigrants, residing in the city of Antofagasta, Chile. To evaluate acculturation, the Revised Acculturation Attitudes Scale by Basabe, Zlovina and Paez was used, and for the mental health assessment, the Self-Assessment Questionnaire OQ-45.2 was applied. Findings show that separation was the most commonly used strategy by Colombians, while in the Peruvian population it was assimilation. Similarly, the Colombian population reported a greater presence of symptoms of mental health problems in all dimensions and in the overall scale than the Peruvian population. In the overall sample, the individuals who used assimilation as their acculturation strategy tended to report more symptoms and difficulties in interpersonal relationships. The data provide evidence that the acculturation strategies used result in a greater or lesser presence of psychopathological symptoms.

Key words: mental health, acculturation, immigration.
\end{abstract}

\footnotetext{
* Dr. Alfonso Urzúa, Escuela de Psicología, Universidad Católica del Norte. Avenida Angamos 0610. Antofagasta, Chile. Fono +56552355822. alurzua@ucn.cl

Esta investigación fue financiada por aportes de la Comisión Nacional de Ciencia y Tecnología del Gobierno de Chile - CONICYT, a través del proyecto FONDECYT 1140843
} 


\title{
Saúde Mental e Estratégias de Aculturaçáo em Imigrantes Colombianos e Peruanos no Norte do Chile
}

\begin{abstract}
Resumo
Mediante um desenho não experimental de tipo transversal contrastou-se a hipótese de que o estado de saúde mental dos imigrantes avaliados difere segundo a estratégia de aculturação que utilizem. Para isso, avaliou-se a diferença média em sintomatologia, papel social e relações interpessoais entre os participantes agrupados por tipo de estratégia de aculturação (integração ou biculturalidade, separação, isolamento e assimilação) numa amostra de 431 imigrantes peruanos e colombianos residentes na cidade de Antofagasta, Chile. Para avaliar a aculturação utilizou-se a Escala revisada de atitudes com relação à aculturação de Basabe, Zlovina e Páez, e para avaliar a Saúde Mental aplicou-se o questionário de Autoavaliação OQ-45.2. Como resultado verificou-se que a estratégia mais utilizada pelos colombianos foi a separação, e pelos peruanos, a assimilação, além de que os primeiros apresentaram maior presença de sintomas de problemas de saúde mental em todas as dimensões e na escala total, em comparação com a população peruana. Na amostra total, os indivíduos que utilizaram a estratégia assimilação tiveram uma maior presença de sintomatologia e dificuldades nas relações interpessoais. Os dados contribuem com evidência com relação a que as estratégias de aculturação utilizadas incidiriam numa maior ou menor presença de sintomatologia psicopatológica.

Palavras-chave: saúde mental, aculturação, imigração.
\end{abstract}

\section{INTRODUCCIÓN}

Se entiende por migración al movimiento de una población hacia el territorio de otro Estado, o dentro del mismo, sea cual fuere su tamaño, su composición o sus causas (OIM, 2006). En los últimos años, dada la percepción de estabilidad económica y de seguridad, Chile se ha convertido en un destino de migración para personas y familias, principalmente de países vecinos limítrofes o sudamericanos como Perú, Ecuador y Colombia, que buscan oportunidades y condiciones que no han encontrado en su país de origen (CEPAL/CELADE, 2006; Stefoni, 2011). Esta inmigración se caracteriza por ser fronteriza, pues cerca del $57 \%$ del total de la población migrante durante el periodo 2005-2014 fue de países como Perú (31,7\%), Argentina (16,3\%) y Bolivia $(8,8 \%)$; y por ser sudamericana $(73 \%$ en el mismo periodo si se incluye a Colombia, Ecuador y Brasil) (DEM, 2016).

La migración es considerada como un proceso que conlleva tanto factores causales y de desarrollo, como consecuencias para la sociedad de origen y para la de destino (Cano \& Soffia, 2009); la persona que se desplaza tiene que disponer de recursos psíquicos y cognitivos para afrontar los cambios que se presentan en el entorno con el fin de adaptarse a su nueva situación. Asimismo, migrar puede constituirse como una instancia de crecimiento y desarrollo, así implique al mismo tiempo experimentar situaciones difíciles y problemáticas (Achotegui, 2010, 2012; Urzúa et al., 2015), como enfrentarse a la adaptación cultural, a la xenofobia, a dificultades de acceso a instituciones públicas, a trámites de regularización migratoria o a dificultades económicas, entre otras; situaciones que pueden tornarse estresantes y alterar el bienestar psíquico de los migrantes al incidir directamente en su salud mental.

La relación entre migración y salud mental se ha estudiado desde el contexto previo al desplazamiento territorial, hasta el momento del establecimiento en el nuevo país, pues variables que se presentan en el lugar de origen como el desempleo, la escasez de recursos económicos o la baja escolaridad, entre otras, actuarían como factores de riesgo para presentar alguna psicopatología tras la migración (Levecque, Lodewyckx \& Vranken, 2007). También, padecer trastornos de ansiedad puede ser relevante e influir al momento de optar por migrar a otro país, pues el proceso de migración podría propiciar la aparición de trastornos de ansiedad y del estado de ánimo (Breslau et al., 2007), como con la aparición de síntomas depresivos en cualquier edad (Sternberg \& Lee, 2013).

Por otro lado, investigaciones recientes presentan hallazgos que sugieren que no existe una relación directa entre el hecho de migrar y la presencia de alguna psicopatología. Por ejemplo, en comparación con los nacidos en EEUU, en los inmigrantes del mismo país se encontraron menos casos de trastornos del estado de ánimo, de ansiedad y de abuso o dependencia de drogas (Alegría et al., 2007; Breslau \& Chang, 2006; Grant et al., 2004; Takeuchi et al., 2007); o también, en un estudio en inmigrantes peruanos en Chile se encontró que el tiempo de permanencia en el nuevo país no está vinculado con la presencia de algún cuadro psiquiátrico (Hospital Clínico Universidad de Chile, 2008).

La relación entre migración y salud mental no es simple, pues el solo hecho de migrar no puede explicar ni predecir la presencia de trastornos psicopatológicos ya que existen 
múltiples variables del individuo y del contexto que actúan como factores mediadores y moderadores (Collazos, Qureshi, Antonín \& Tomás-Sábado, 2008). Además, hay que tener en cuenta que migrar requiere de un buen estado de salud físico y mental para poder realizar adecuadamente la serie de cambios que implican el asentamiento, la adaptación y la integración (Tizón, 1994).

Una de las variables estudiadas que puede considerarse como el contexto en el que se presenta la relación entre salud mental y migración es el llamado proceso de aculturación, definido por Berry (2005) como el cambio cultural que ocurre en el encuentro entre grupos sociales con diferentes costumbres, normas e ideologías, ante las cuales pueden presentarse modificaciones reactivas, como el rechazo por o hacia la nueva cultura; o indirectas, como en los procesos psicológicos que pueden incidir en la adaptación a la nueva sociedad. Al entrar en contacto con la nueva cultura, el migrante inicia una serie de cambios internos y en su repertorio conductual, proceso definido por Graves (1967) como aculturación psicológica, el cual se concreta en la praxis con las llamadas estrategias de aculturación.

Según Berry (2001), las estrategias de aculturación se rigen por el deseo de mantener la idiosincrasia cultural y el interés de participar en la nueva cultura. Desde este modelo, y con el Modelo Interactivo de Aculturación (MIA) propuesto por Bourhis, Moise, Perreault y Senecal (1997, en Fajardo, Patiño \& Patiño, 2008), existen cuatro mecanismos de aculturación: (a) la integración (también llamada biculturalidad), en donde el migrante busca mantener sus valores de origen y al mismo tiempo participar en la nueva cultura; (b) la asimilación, en la cual opta por asumir los valores de la nueva cultura y renuncia a la tradicional y autóctona; (c) la marginalización, en donde no busca interactuar con la nueva cultura ni mantener la de origen; y (d) la separación, con la que pretende validar y mantener solo la tradición de su propia cultura al establecer casi nulos contactos con la nueva sociedad (Berry, 2001; Sánchez \& López, 2008).

Según el camino que se opte seguir en este proceso de aculturación, que más que una decisión es una negociación entre los componentes del contexto cultural de acogida y las herramientas y competencias propias del inmigrante, la estrategia empleada va a influir en el estado de salud mental (Yáñez \& Cárdenas, 2010). Por ejemplo, en una investigación con mujeres latinas inmigrantes que viven en EEUU se demostró que la aculturación genera mayor nivel de estrés, pues al lidiar con problemas como la adaptación cultural, la situación legal, el sistema institucional que las oprime, la discriminación social y la dificultad económica, existe un mayor riesgo de que presenten síntomas depresivos que alteren su nivel de salud mental en comparación con latinas nacidas en dicho país (Sternberg \& Lee, 2013). Por otro lado, en estudios en Chile se ha demostrado que los altos niveles de estrés por aculturación inciden en el aumento de sintomatología asociada a problemas de salud mental (Urzúa, Heredia \& Caqueo-Urízar, 2016).

Teniendo en cuenta que la evidencia no es del todo concluyente, para el presente estudio surgió el interrogante: "¿incide en la salud mental de adultos inmigrantes la estrategia de aculturación utilizada?" Para dar respuesta a la pregunta se planteó como objetivo analizar la relación existente entre la salud mental y las estrategias de aculturación utilizadas en la población inmigrante.

Como hipótesis se tiene que el tipo de estrategia utilizada en el encuentro con la nueva cultura influye en la salud mental del inmigrante; $y$, específicamente, que la presencia de la sintomatología asociada a problemas de salud mental estaría relacionada con el uso de estrategias de aculturación de marginalización o separación, y no con estrategias de integración o asimilación.

\section{MÉTODO}

\section{Muestra}

En este estudio participaron 431 inmigrantes peruanos y colombianos mayores de 18 años que habían residido en la ciudad de Antofagasta durante al menos seis meses (esto para excluir de la muestra a extranjeros visitantes o turistas).

Tabla 1

Distribución de resultados por sexo y edad en población peruana y colombiana

\begin{tabular}{|c|c|c|c|c|c|c|c|c|c|c|c|c|}
\hline & \multicolumn{6}{|c|}{ Sexo } & \multicolumn{6}{|l|}{ Edad } \\
\hline & \multicolumn{2}{|c|}{ Hombre } & \multicolumn{2}{|c|}{ Mujer } & \multirow{2}{*}{$\frac{\text { Total }}{\mathrm{N}}$} & \multirow{2}{*}{$\begin{array}{l}\% \\
\%\end{array}$} & \multicolumn{2}{|c|}{ Hombres } & \multicolumn{2}{|c|}{ Mujeres } & \multicolumn{2}{|l|}{ Total } \\
\hline & $\mathrm{n}$ & $\%$ & $\mathrm{n}$ & $\%$ & & & $\mathrm{M}$ & $\mathrm{DE}$ & $\mathrm{M}$ & $\mathrm{DE}$ & M & $\mathrm{DE}$ \\
\hline Colombianos & 111 & 47.80 & 121 & 52.20 & 232 & 53.80 & 32.59 & 8.67 & 32.40 & 9.65 & 32.49 & 9.17 \\
\hline Peruanos & 96 & 48.20 & 103 & 51.80 & 199 & 46.20 & 33.40 & 9.77 & 34.88 & 9.83 & 34.16 & 9.80 \\
\hline Total & 207 & 48.00 & 224 & 52.00 & 431 & 100 & 32.97 & 9.18 & 33.53 & 9.79 & 33.26 & 9.49 \\
\hline
\end{tabular}


Se eligieron colombianos y peruanos por ser el grupo más grande de inmigrantes en esta ciudad (DEM, 2015); atraídos principalmente por la minería y los servicios derivados de ella, como la construcción, el comercio y las ventas.

De estos, 207 fueron hombres (48\%) y 224, mujeres (52\%); su edad fluctuó entre los 18 y los 65 años; el tiempo de permanencia promedio de los colombianos encuestados fue de 2.3 años, y el de los peruanos, 4 años; cerca del $30 \%$ de ambas muestras demostró estudios secundarios terminados; y un $20 \%$ de colombianos y $14 \%$ de peruanos contaba con formación técnica completa. Por otro lado, el $75 \%$ en ambos grupos declaró ser activo laboralmente; el $48 \%$ de colombianos y $44 \%$ de peruanos declaró ingresos entre 100000 y 300000 CLP (entre 150 y 450 USD), y $31 \%$ de colombianos y $38 \%$ de peruanos entre 300000 y 600000 CLP (entre 450 y 900 USD). La distribución de los encuestados por sexo y edad se muestra en la Tabla 1.

\section{Instrumentos}

Los instrumentos utilizados fueron la Escala de actitudes hacia la aculturación (Basabe, Zlovina \& Páez, 2004) y el Cuestionario de autoevaluación OQ-45.2 (Von Bergen \& De La Parra, 2002).

Escala de actitudes hacia la aculturación. Este cuestionario sobre estrategias y actitudes de aculturación elaborado por Basabe, Zlovina y Páez (2004) a partir de lo propuesto por Berry (2001) consta de dos subescalas que miden, por un lado, el deseo de mantener las costumbres de la sociedad de origen y, por otro, el de adoptar las costumbres del país de acogida. Cada una de estas subescalas se compone de 6 ítems que abordan lo privado (tres preguntas que apuntan a conocer costumbres en relación a la familia, las amistades y las creencias) y lo público (tres preguntas orientadas a conocer las costumbres vinculadas al trabajo, al sistema de bienestar social, y al sistema político de gobierno). La forma de dar respuesta al cuestionario es marcando de 1 (nada) a 5 (mucho), según corresponda en cada enunciado. Esta escala ha sido utilizada en estudios previos en migrantes en Chile (Arenas \& Urzúa, 2016) y en otros estudios latinoamericanos con una población similar (Castro, 2011; Sosa \& Zubieta, 2012), sin registrarse información sobre sus propiedades psicométricas. En el presente estudio se obtuvieron los siguientes valores de confiabilidad mediante el estadístico alfa de Cronbach: .81 para mantener costumbres del país de origen, .65 para adoptar costumbres del país de llegada, .81 para interés por mantener costumbres y .82 para interés por adoptar costumbres.

Cuestionario de Autoevaluación OQ-45.2. Este instrumento mide el progreso de la terapia psicológica al evaluar el nivel de salud mental del paciente durante la misma, y cuenta con indicadores de consistencia superiores a .75 , validez concurrente, validez de criterio, sensibilidad de .90 (proporción de verdaderos positivos correctamente identificados) y especificidad de .93 (proporción de verdaderos negativos correctamente identificados) (Von Bergen \& De La Parra, 2002). Es una escala sumativa tipo likert, de nivel ordinal, a la que se responde optando entre cinco categorías de acuerdo a qué tanto le ocurre lo señalado en cada ítem: "Nunca $=0$ ", "Casi nunca $=1$ ", "A veces $=2$ ", "Con frecuencia $=3$ " y "Casi siempre $=4 "$ ". Y está compuesta por tres dimensiones: Síntomas (SD), con 25 ítems para detectar estados depresivos y/o ansiosos; Relaciones Interpersonales (RI), con 11 ítems para medir grado de satisfacción y problemas en esta área; y Rol social (SR), con 9 ítems que miden los niveles de conflicto, insatisfacción, trastorno o inadecuación de la persona en relación al empleo, los roles familiares y el ocio. Los puntajes se obtienen sumando el total de puntuaciones en la escala completa (valores de 0 a 4 para cada ítem), cuyo rango va de 0 a 180 puntos, y sumando los puntajes en cada dimensión. El puntaje de corte de la escala total es de 73, el cual divide entre puntaje bajo y alto y alude al grado de incomodidad en su vida en general, considerando síntomas, relaciones interpersonales y rol social; para estas dimensiones el punto de corte es de 43, 16 y 14 respectivamente.

\section{Procedimiento}

Para llevar a cabo esta investigación se contó con la aprobación de los comités de Ética de la Universidad Católica del Norte y de la Comisión Nacional de Ciencia y Tecnología (CONICYT).

Como no se conocía la cantidad exacta del universo poblacional ni del marco de muestreo, se utilizó uno de tipo no probabilístico a través de la estrategia de bola de nieve, en donde se eligieron aleatoriamente los primeros participantes con un máximo de 6 cadenas para lograr una adecuada representatividad. Se contactó a estas personas para invitarlos a participar de manera voluntaria en el estudio y se les explicó el objetivo de investigación para que luego firmaran el consentimiento informado, en donde sugerían tres contactos como posibles participantes. Si alguno de ellos se negaba a participar o si ya estaba en la muestra, dicha cadena se rompía.

El tiempo de aplicación de los instrumentos fue de aproximadamente dos horas, y ocho de cada diez personas a las que se les solicitó la participación llenaron la encuesta (grado de participación del 80\%). Los datos fueron ingresados en una base construida en el software SPSS 17.0.

\section{Análisis de resultados}

Para evaluar las diferencias de las medias entre las variables dadas por el sexo y por el país de origen se 
utilizó la prueba $T$ de student. Posteriormente se realizó un ANOVA para evaluar posibles diferencias en las medias en salud mental entre las distintas estrategias de aculturación. Se realizaron pruebas de normalidad a las variables (Kolmogorov-Smirnov y Shapiro-Wilk), sin embargo, pese a que algunas de ellas no presentaron una distribución normal con tamaños muestrales por encima de 40 , la prueba $\mathrm{T}$ y el ANOVA pudieron utilizarse a pesar de que la distribución de los datos fuera asimétrica y no existieran valores atípicos (Pardo, Ruiz \& San Martín, 2014).

\section{RESULTADOS}

A continuación se presentan los estadísticos descriptivos de las variables estrategia de aculturación y salud mental; posteriormente se muestra la puntuación media en salud mental de los participantes categorizados según la estrategia utilizada; y, finalmente, se da cuenta de los resultados del ANOVA.

\section{Estrategia de aculturación}

A nivel de la muestra total, la estrategia de aculturación más utilizada fue la de integración (también llamada bicultutalidad), seguida por la de asimilación. Al estratificar el análisis por sexo, en los hombres se mantuvo la integración como la estrategia más utilizada, mientras que en las mujeres fue la asimilación, seguida por la separación (véase Tabla 2).

En el análisis por país se observa que la estrategia más utilizada por la población colombiana es la de separación, seguida por la integración; mientras que en los peruanos es la asimilación, seguida por la integración (véase Tabla 3).

\section{Salud mental}

A nivel de la muestra general, las mujeres presentan mayores puntajes que los hombres en todos los dominios evaluados y en la escala total; sin embargo, esta diferencia no es estadísticamente significativa para ninguna de las dimensiones evaluadas (véase Tabla 4).

$\mathrm{Al}$ analizar las medias dadas según el país de origen se observa que la población colombiana reporta puntajes mayores que la peruana en todas las dimensiones y en la escala total (véase Tabla 5), pero solo en la subescala de relaciones personales la probabilidad de encontrar el valor de Tes menor a .05. Por esta razón se rechaza la hipótesis nula de que no hay diferencias significativas entre las medias, teniendo en cuenta que la media de los colombianos es significativamente mayor que la de los peruanos $\left(\mathrm{t}_{(407)}=4.666 ; \mathrm{p}=.000\right)$.

En la Tabla 6 se muestra la media y desviación estándar en cada una de las dimensiones de la salud mental evaluadas, divididas tanto en categorías de estrategias de aculturación como por país.

Tabla 2

Estrategias de aculturación mayormente utilizadas, muestra total y por sexo

\begin{tabular}{lllllll}
\hline & Hombres & \multicolumn{3}{c}{ Mujeres } & Total \\
& $\mathrm{n}$ & $\%$ & $\mathrm{~N}$ & $\%$ & $\mathrm{~N}$ & $\%$ \\
\hline Integrados (biculturales) & 63 & 31.2 & 58 & 26.1 & 121 & 28.5 \\
Separados & 40 & 19.8 & 61 & 27.5 & 101 & 23.8 \\
Asimilados & 52 & 25.7 & 62 & 27.9 & 114 & 26.9 \\
Marginados & 47 & 23.3 & 41 & 18.5 & 88 & 20.8 \\
\hline
\end{tabular}

Tabla 3

Distribución porcentual variable estrategias de aculturación por sexo y país

\begin{tabular}{|c|c|c|c|c|c|c|c|c|c|c|c|c|}
\hline & \multicolumn{6}{|c|}{$\mathrm{COL}$} & \multicolumn{6}{|l|}{$\mathrm{PE}$} \\
\hline & \multicolumn{2}{|c|}{ Hombres } & \multicolumn{2}{|c|}{ Mujeres } & \multicolumn{2}{|c|}{ Total } & \multicolumn{2}{|c|}{ Hombres } & \multicolumn{2}{|c|}{ Mujeres } & \multicolumn{2}{|c|}{ Total } \\
\hline & $\mathrm{n}$ & $\%$ & $\mathrm{n}$ & $\%$ & $\mathrm{n}$ & $\%$ & $\mathrm{n}$ & $\%$ & $\mathrm{n}$ & $\%$ & $\mathrm{n}$ & $\%$ \\
\hline Integrados (biculturales) & 33 & 31.1 & 30 & 25.2 & 63 & 28.0 & 30 & 31.3 & 28 & 27.2 & 58 & 29.1 \\
\hline Separados & 22 & 20.8 & 43 & 35.5 & 65 & 28.9 & 18 & 18.8 & 18 & 17.5 & 36 & 18.1 \\
\hline Asimilados & 27 & 25.5 & 23 & 19.3 & 50 & 22.2 & 25 & 26 & 39 & 37.9 & 64 & 32.2 \\
\hline Marginados & 24 & 22.6 & 23 & 19.3 & 47 & 20.9 & 23 & 24 & 18 & 17.5 & 41 & 20.6 \\
\hline
\end{tabular}

Nota. $\mathrm{PE}=$ población peruana; $\mathrm{COL}$ : población colombiana. 
Tabla 4

Medias y DE en dominios de salud mental evaluados por sexo y total

\begin{tabular}{lllllll}
\hline & Hombres & \multicolumn{3}{c}{ Mujeres } & \multicolumn{3}{c}{ Total } \\
& M & DE & M & DE & M & DE \\
\hline Sintomatología (1) & 31.96 & 11.43 & 33.78 & 11.11 & 32.90 & 11.30 \\
Rol Social (2) & 15.96 & 3.69 & 16.25 & 3.26 & 16.12 & 3.47 \\
Relaciones Interpersonales (3) & 23.28 & 4.46 & 23.33 & 4.42 & 23.31 & 4.44 \\
Escala Total (4) & 71.31 & 15.32 & 73.53 & 14.21 & 72.45 & 14.79 \\
\hline
\end{tabular}

Nota. Diferencias entre hombres y mujeres no significativas $(1)\left(\mathrm{t}_{(403)}=-1.626 ; \mathrm{p}=.105\right) ;(2)\left(\mathrm{t}_{(407)}=-1.111 ; \mathrm{p}=.911\right)$; (3) $\left(\mathrm{t}_{(416)}=-.858 ; \mathrm{p}=.392\right) ;(4)\left(\mathrm{t}_{(388)}=-1.483 ; \mathrm{p}=.139\right)$.

Tabla 5

Medias y DE en dimensiones de salud mental evaluados por sexo y país

\begin{tabular}{|c|c|c|c|c|c|c|c|c|c|c|c|c|}
\hline & \multicolumn{6}{|l|}{$\mathrm{COL}$} & \multicolumn{6}{|l|}{$\mathrm{PE}$} \\
\hline & \multicolumn{2}{|c|}{ Hombres } & \multicolumn{2}{|c|}{ Mujeres } & \multicolumn{2}{|l|}{ Total } & \multicolumn{2}{|c|}{ Hombres } & \multicolumn{2}{|c|}{ Mujeres } & \multicolumn{2}{|l|}{ Total } \\
\hline & M & $\mathrm{DE}$ & M & $\mathrm{DE}$ & M & $\mathrm{DE}$ & M & $\mathrm{DE}$ & M & $\mathrm{DE}$ & M & $\mathrm{DE}$ \\
\hline Sintomatología & 32.36 & 12.41 & 33.54 & 12.24 & 32.97 & 12.30 & 31.50 & 10.26 & 34.07 & 9.74 & 32.81 & 10.06 \\
\hline Rol Social & 16.22 & 3.68 & 16.35 & 3.59 & 16.29 & 3.63 & 15.67 & 3.69 & 16.15 & 2.85 & 15.91 & 3.29 \\
\hline $\begin{array}{l}\text { Relaciones Inter- } \\
\text { personales }\end{array}$ & 24.07 & 4.52 & 24.37 & 4.48 & 24.22 & 4.49 & 22.38 & 4.24 & 22.06 & 4.02 & 22.21 & 4.13 \\
\hline Escala Total & 73.04 & 15.70 & 74.47 & 15.30 & 73.78 & 15.46 & 69.30 & 14.74 & 72.35 & 12.74 & 70.84 & 13.80 \\
\hline
\end{tabular}

Nota. $\mathrm{PE}=$ población peruana; COL: población colombiana.

En la dimensión de sintomatología se observa una mayor presencia de sintomatología en aquellos que optan por una estrategia de asimilación, mientras que los integrados (o biculturales) son los que reportan menos síntomas. Sin embargo, estas solo son estadísticamente significativas en la muestra total $\left(\mathrm{F}_{(3,401)}=4.243 ; \mathrm{p}=.006\right)$ y en la población colombiana $\left(\mathrm{F}_{(3,213)}=4.707 ; \mathrm{p}=.003\right)$, en donde los que utilizan la estrategia de marginación tienen una media significativamente mayor que los separados $(\mathrm{p}=.004 \mathrm{y}$ $\mathrm{p}=.001$ respectivamente).

En las relaciones interpersonales, los asimilados son quienes reportan la mayor media en dificultades, mientras que los marginados, la menor $(\mathrm{p}=.012)$. Esta diferencia solo es significativa a nivel de la muestra total $\left(\mathrm{F}_{(3,405)}=3.648\right.$; $\mathrm{p}=0.013$ ); y no se observan otras diferencias significativas al separar la muestra por país.

Finalmente, tanto en la dimensión del rol social como en la escala total, los asimilados son los que presentan mayor puntaje por sobre los que utilizan otras estrategias; sin embargo, no se encuentran diferencias estadísticamente significativas entre los puntajes de cada estrategia, en la muestra total, ni al estratificar el análisis por país.

\section{DISCUSIÓN}

De acuerdo con los resultados es posible identificar factores comunes y propios de cada grupo migratorio. Ambas poblaciones utilizan la estrategia de integración (o biculturalidad), sin embargo no es la más usada, dado que en el caso de la población colombiana la mayormente reportada es la de separación, seguida por la integración, mientras que en los peruanos es la asimilación, seguida por la integración. En el caso de la salud mental, la población colombiana reporta peores resultados en comparación con la peruana, especialmente en la dimensión de relaciones interpersonales.

En la hipótesis inicial de este estudio se planteaba que la salud mental del inmigrante estaría influida por el tipo de estrategia utilizada en el encuentro con la nueva cultura; y que, específicamente, la presencia de sintomatología psicopatológica asociada a problemas de salud mental estaría relacionada con la presencia de las estrategias de aculturación de marginalización y separación, a diferencia de las estrategias de integración y asimilación. Según los resultados encontrados, estos difieren parcialmente de lo 
Tabla 6

Medias en Salud Mental según Estrategia de Aculturación y Nacionalidad

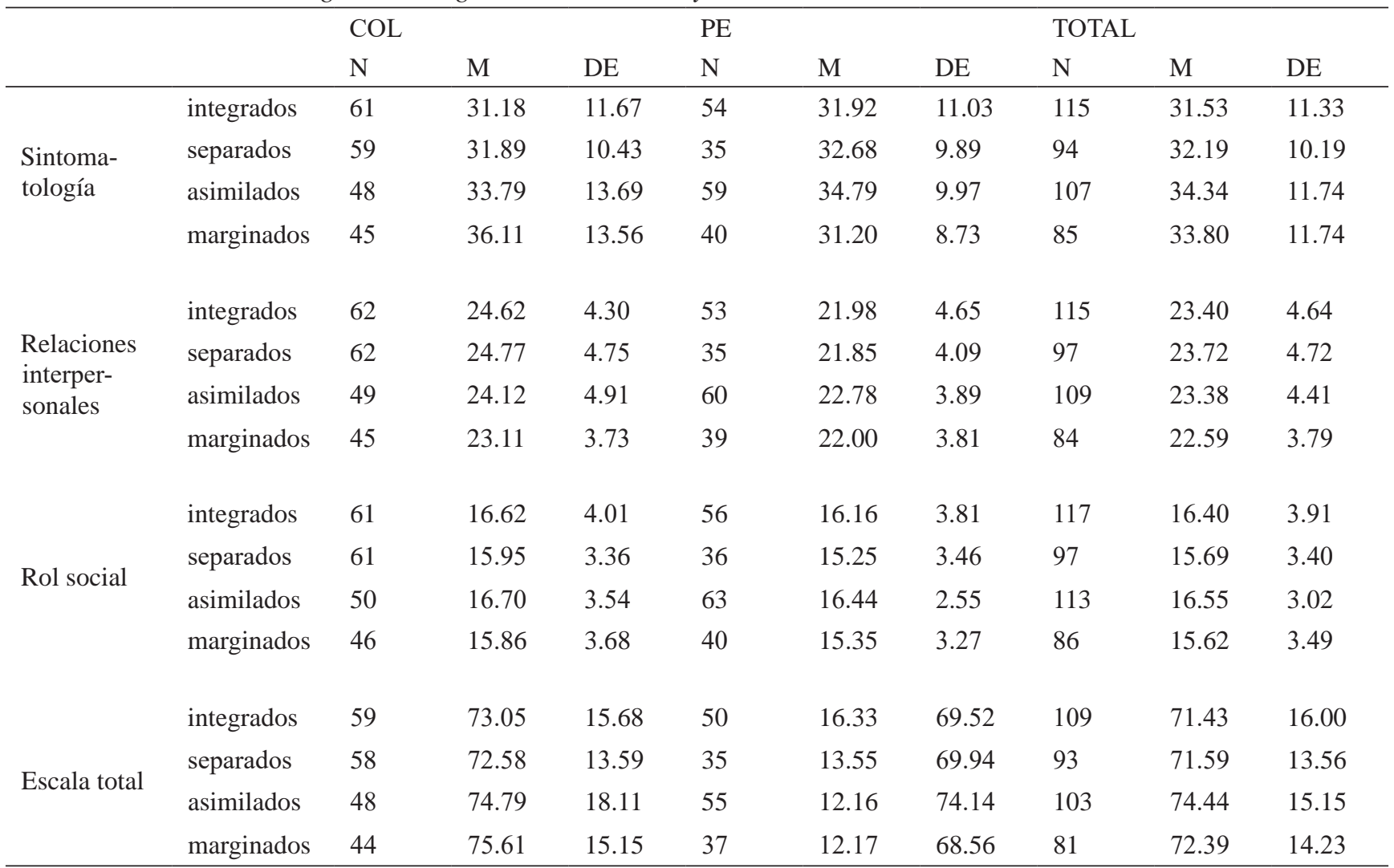

Nota. $\mathrm{PE}=$ población peruana; $\mathrm{COL}$ : población colombiana.

esperado pues se observa una mayor sintomatología declarada en aquellos que optan por la asimilación y menos en los que utilizan la integración o biculturación (especialmente en los colombianos). De igual manera, son los asimilados quienes presentan mayores problemas en sus relaciones interpersonales.

En cuanto a la salud mental, los migrantes experimentan una incomodidad general con su nueva vida, siendo particularmente evidente la situación con respecto a las relaciones sociales que establecen tanto en términos familiares como en cuanto a los ámbitos laborales, escolares, comunitarios y sociales, lo cual puede estar ligado con la dificultad de ajustarse a los roles que se adquieren en dichos ámbitos. Particularmente, las mujeres manifiestan que experimentan mayor presencia de síntomas de ansiedad y depresión, mayor conflicto en las relaciones interpersonales y mayor dificultad en su capacidad de ajuste en los roles que asumen, y por tanto tienden a manifestar o percibir una grado mayor de incomodidad en el modo de ir construyendo su vida en la nueva sociedad chilena, específicamente en el contexto de la ciudad de Antofagasta. A esto se le suma que el contexto de las familias colombianas y peruanas en sus respectivos países de origen da cuenta de una inseguridad económica, política y social que no permiten obtener la estabilidad anhelada para la sobrevivencia de las personas (Stefoni, 2004). Tal como se evidencia en el estudio de Levecque, Lodewyckx y Vranken (2007), las condiciones desfavorables en el empleo, ingresos económicos y escolaridad, entre otros, se presentan como factores de riesgo en términos de salud mental en las personas.

Al tomar la decisión de emigrar hacia otro país se dispone de una nueva forma de solucionar estas problemáticas y se elabora un conjunto de expectativas con respecto al encuentro con esta nueva y diferente cultura. Con esto se aspira a encontrar nuevas y mejores oportunidades laborales, un sistema social menos agresivo y peligroso, y un mejor contexto social, cultural, económico y político que permita el desarrollo y crecimiento saludable de los individuos y de las familias, mediante una mayor flexibilización de los espacios de interacción; sin embargo, esto no llega tan rápidamente ni tan fácilmente como se espera, lo que convierte a la brecha entre lo real y lo esperado en un factor de riesgo para su salud mental. 
Con respecto a las estrategias de aculturación de los inmigrantes peruanos y colombianos en su encuentro con esta nueva cultura chilena/antofagastina, existe una mayor inconformidad con la vida en general en quienes han optado por ser asimilados por esta cultura, dejando así a un lado sus tradiciones y costumbres de origen. Aun cuando no se tiene evidencia al respecto, esto se podría explicar por la existencia de una gran resistencia del grupo de acogida, dado que implica una nueva entrada al sistema, la cual tiende a romper el orden y la estabilidad necesaria para mantener la propia existencia con base en su sentido de identidad como nativos chilenos y antofagastinos. Por esto se repele cualquier intento de perder dicha configuración, lo cual incide en la sensación de incomodidad de los inmigrantes asimilados, quienes presentan principalmente dificultad en las relaciones interpersonales y en las funciones que asumen en este nuevo contexto, tales como el rol en el trabajo, en la familia, en el sistema escolar y en las diversas áreas de interacción. En menor grado se aprecia este malestar en quienes utilizan la integración (o biculturalidad) como estrategia de aculturación; estos dos mecanismos son los que más se utilizan en el grupo de inmigrantes en el contexto de la sociedad de Antofagasta.

Por otro lado, quienes manifiestan sentir menor grado de insatisfacción con el modo de vida establecido son el grupo que se rige por la separación. Esto puede analizarse en términos sistémicos teniendo en cuenta que posiblemente no hay esfuerzos por intentar ser aceptado e integrado en la cultura de acogida, y por ende no recibe el rechazo desde el sistema social antofagastino, pues existe un menor grado de interacción y porque su sentido de pertenencia e identidad está vinculado al contacto permanente con sus propios connacionales, cuyo sistema es el que se intenta mantener en equilibrio; con ello se intenta asegurar la sobrevivencia del grupo que está inmerso en la cultura de llegada.

A este grupo le siguen quienes deciden escoger la marginación como estrategia de aculturación. En esta puede existir la motivación de no sentirse parte de ninguno de los sistemas culturales y sociales dado que el ser reconocido como peruano o colombiano implica interacciones disfuncionales con la población chilena, situación que distancia al inmigrante de la posibilidad de encontrar el bienestar psicológico, económico, laboral y familiar por el cual se decidió emigrar, y en donde el sentido de ello está dado principalmente por el proporcionar a su familia de origen la gratificación de sus principales necesidades, así se encuentre distanciado de ella. De esto da cuenta Zapata (2009) en el estudio sobre familias transnacionales en el que se advierte cómo los padres y madres inmigrantes logran cumplir su rol parental y compensar la distancia física mediante los recursos de comunicación disponibles como el teléfono o el internet, entre otros (Pedone, 2007).

El rol social es definido en el contexto de la relación con otros, en la interacción social y en el encuentro con otras subjetividades; así como plantean Carabaña y Lamo de Espinoza (1978), la vida en sociedad conlleva un proceso en el que a través de la interacción surgen símbolos que se internalizan y regulan los estímulos que provienen del entorno y sus propias respuestas; por tanto, es un proceso que permite dar sentido a la propia definición del sí mismo. En este sentido, al ocurrir un acto social, el individuo asume un conjunto de actitudes que es valorado por los otros en virtud de la situación que amerita, y en este sentido, la tendencia a la renuncia de los símbolos, valores y tradiciones de la cultura de origen puede ser el resultado de la inserción en el nuevo grupo social, la cual conlleva un quiebre, una pérdida y un duelo con respecto a lo que se pierde; y que puede resultar en un sentimiento de malestar, incomodidad y dificultad, pues se deja de ser alguien para asumir otra identidad distinta, aunque esto implique el rechazo de la sociedad chilena antofagastina, siendo esta resistente a la integración al tener límites rígidos y al establecer barreras para dificultar la toma de roles sociales por parte de los inmigrantes.

Asimismo, la situación del inmigrante se complejiza dado que al asentarse en la sociedad antofagastina con un alto grado de vulnerabilidad en cuanto su salud mental, y al enfrentarse con los límites altamente rígidos de los diversos subsistemas sociales, es posible que se detonen conductas o estados sintomáticos.

Cabe destacar la necesidad urgente de considerar la permanencia de los hábitos y costumbres del país de origen en una sana convivencia con los del país receptor, tanto en las acciones, estrategias y políticas públicas para la integración como en la labor social que promueva el bienestar social entre las comunidades receptoras y migrantes, ya que la completa eliminación de la identidad del otro minoritario (cuando es asimilado), constituye un riesgo para su bienestar y salud mental.

\section{REFERENCIAS}

Achotegui, J., (2010). Cómo evaluar el estrés y el duelo migratorio. Llançà, España. El mundo de la Mente.

Achotegui, J. (2012). La crisis como factor agravante del Síndrome de Ulises (Síndrome del Duelo Migratorio Extremo). Revista Temas de Psicoanálisis, 3, 1-16.

Alegría, M., Mulvaney-Day, N., Torres, M., Polo, A., Cao, Z., y Canino, G. (2007). Prevalence of psychiatric disorders across Latino subgroups in the United States. American 
Journal of Public Health, 97(1), 68-75. Doi: http://dx.doi. org/10.2105/AJPH.2006.087205

Arenas, P., Urzúa, A., (2016) Estrategias de Aculturación e identidad étnica. Un estudio en migrantes Sur-Sur en el norte de Chile. Universitas Psychologica, 15(1), 117-128.

Basabe, N. Zlovina, A, Paez, D. (2004). Integración sociocultural y adaptación psicológica de los inmigrantes extranjeros en el País Vasco. País Vasco: Eusko Jaurlaritzaren Argitalpen Zerbitzu Nagusia.

Berry, J.W., (2001). A Psychology of Immigration. Journal of Social Issues, 57(3), 615-631. Doi: http://dx.doi. org/10.1111/0022-4537.00231

Berry, J. (2005). Acculturation: Living successfully in two cultures. International Journal of Intercultural Relations, 26(6), 697-712.

Breslau, J., Aguilar-Gaxiola, S., Borges, G., Castilla-Puentes, R. C., Kendler, K. S., Medina-Mora, M. E., y cols. (2007). Mental disorders among English-speaking Mexican immigrants to the US compared to a national sample of Mexicans. Psychiatry Research, 15(1-2), 115-122. Doi: http:// dx.doi.org/10.1016/j.psychres.2006.09.011

Breslau, J., y Chang, D. F. (2006). Psychiatric disorders among foreign-born and US-born Asian-Americans in a US national survey. Social Psychiatry and Psychiatric Epidemiology, 41(12), 943-950. Doi: http://dx.doi. org/10.1007\%2Fs00127-006-0119-2

Cano, V. y Soffia, M. (2009). Los estudios sobre migración internacional en Chile: apuntes y comentarios para una agenda de investigación actualizada. Papeles de población, 15(61), 129-167.

Carabaña, J., Lamo de Espinoza, E. (1978). La teoría Social del Interaccionismo Simbólico. Revista Española de Investigaciones Sociológicas, 1, 159-204.

Castro, A. (2011). Estrategias de aculturación y adaptación psicológica y sociocultural de estudiantes extranjeros en la Argentina. Interdisciplinaria, 28, 115-130.

CEPAL/CELADE (2006). Migración Internacional. Observatorio Demográfico. América Latina y el Caribe. $\mathrm{N}^{\circ} 1$ Abril, 2006. Santiago, Chile.

Collazos, F., Qureshi, A., Antonín, M., y Tomás-Sábado, J. (2008). Estrés aculturativo y salud mental en la población inmigrante. Papeles del Psicólogo, 29(3), 307-315.

DEM. Departamento de Extranjería y Migración del Ministerio del Interior y Seguridad Pública, Gobierno de Chile (2016). Migración en Chile 2005 - 2014. Recuperado de la página en internet del organismo en http://www.extranjeria.gob. $\mathrm{cl} /$ media/2016/02/Anuario-Estad\%C3\%ADstico-NacionalMigraci\%C3\%B3n-en-Chile-2005-2014.pdf

Fajardo, M., Patiño, M.I., \& Patiño, C. (2008). Estudios actuales sobre aculturación y salud mental en inmigrantes: Revisión y perspectivas. Revista Iberoamericana de Psicología: Ciencia y Tecnología, 1, 39-50.
Grant, B. F., Stinson, F. S., Hasin, D. S., Dawson, D. A., Chou, S. P., y Anderson, K. (2004). Immigration and lifetime prevalence of DSM-IV psychiatric disorders among Mexican Americans and non-Hispanic Whites in the United States. Archives of General Psychiatry, 61(12). Doi: http://dx.doi. org/10.1001/archpsyc.61.12.1226

Graves, T. (1967). Psychological acculturation in a tri-ethnic community. South-western Journal of Antrophology, 23, 337-350.

Hospital Clínico Universidad de Chile (2008). Diagnóstico y factibilidad global para la implementación de políticas globales de salud mental para inmigrantes de la zona norte de la región metropolitana. Recuperado de https://www. google.cl/url? sa=t\&rct=j\&q=\&esrc=s\&source=web\&cd=1 $\&$ cad $=$ rja $\&$ ved $=0 \mathrm{CC} 0 \mathrm{QFjAA} \& u r l=h \mathrm{htp} \% 3 \mathrm{~A} \% 2 \mathrm{~F} \% 2 \mathrm{Fweb}$. minsal.cl\%2Fportal\%2Furl\%2Fitem \%2F71 e4f2dd2b60846 0e04001011f01239d.pdf\&ei=jhO8UuL1LuehsASCyYCgA g\&usg=AFQjCNFTscbAQXiKobK-o7XfjjBl-VdFSw\&sig 2=fd0LxLrMTOsst8DyGifE4w\&bvm=bv.58187178,d.cWc

Levecque, K., Lodewyckx, I., \& Vranken, J. (2007). Depression and generalized anxiety in the general population in Belgium: a comparison between native and immigrant groups. Journal of Affective Disorders, 97(1-3), 229-239. DOI: http://dx.doi.org/10.1016/j.jad.2006.06.022

OIM. Oficina Internacional de Migraciones (2006). Derecho internacional sobre migración (Informe $\mathrm{N}^{\circ} 7$ ); Glosario sobre migraciones. Recuperado de la página en internet del organismo en http://publications.iom.int/system/files/pdf/ iml_7_sp.pdf

Pardo, A., Ruiz, MA., San Martín, R. (2014). Análisis de datos en ciencias sociales y de la salud I. Madrid. Editorial Síntesis.

Pedone, C. (2007). Los/as hijos/as de la migración ecuatoriana: lecturas transnacionales de los cambios familiares. Ponencia presentada en el $V$ Congreso sobre la Inmigración en España. Migraciones y Desarrollo Humano, Valencia, España. Universitat De València, Ceim - Centro de Estudio para la Integración Social y Formación de Inmigrantes. Recuperado de: http://www.migrastudium.org/doc/LRecolons $\% 20$ Transnacionalismo $\% 20 \mathrm{y} \% 20$ redes $\% 20$ migratorias\%20VCongreso2007.doc

Sánchez, M. G. \& López, M., M., (2008). Ansiedad y modos de aculturación en la población inmigrante. Apuntes de Psicología Colegio Oficial de Psicología 26 (3), 399-410.

Sosa, F. y Zubieta, E. (2012). Aculturación psicológica y creencias en estudiantes universitarios migrantes y no migrantes. Hologramática, 2(17), 45-70.

Stefoni, C., (2004). Inmigrantes transnacionales: la formación de comunidades y la transformación en ciudadanos. FLACSO, Facultad Latinoamericana de Ciencias Sociales, Sede Chile (9). Recuperado de http://bibliotecavirtual.clacso.org. ar/ar/libros/chile/flacso/artstef.pdf

Stefoni, C. (2011). Perfil Migratorio de Chile. Documento de la Oficina Internacional para las migraciones OIM. Recu- 
perado de http://priem.cl/wp-content/uploads/2015/04/Stefoni_Perfil-Migratorio-de-Chile.pdf.

Sternberg, R. \& Lee, K. (2013). Depressive symptoms of midlife Latinas: effect of immigration and sociodemographic factors. International Journal of Women's Health, 5, 301308. doi: http://dx.doi.org/10.2147/IJWH.S43132

Takeuchi, D. T., Zane, N., Hong, S., Chae, D. H., Gong, F., Gee, G. C., y cols. (2007). Immigration-related factors and mental disorders among Asian Americans. American Journal of Public Health, 97(1), 84-90. Doi: http://dx.doi.org/10.2105/ AJPH.2006.088401

Tizón, J., (1994). Emigrar: sonar, sufrir...tal vez elaborar, tal vez crear. Revista de Treball Social, 136, 22-38.

Urzúa, A., Heredia, O., Caqueo-Urízar, A. (2016) Salud mental y estrés por aculturación en inmigrantes sudamericanos en el norte de Chile. Revista Médica de Chile,144, 563-570
Urzúa, A., Vega, M., Jara, A., Trujillo, S., Muñoz, R. CaqueoUrízar, A. (2015) Calidad de vida percibida en inmigrantes sudamericanos en el norte de Chile. Terapia Psicológica, 33(2), $139-156$.

Von Bergen A, De La Parra G. OQ-45.2 (2002). Cuestionario para Evaluación de Resultados y Evolución en Psicoterapia: Adaptación, Validación e Indicaciones para su Aplicación e Interpretación. Terapia Psicológica, 20, 161-176.

Yáñez, S., Cárdenas, M. (2010). Estrategias de Aculturación, Indicadores de Salud Mental y Bienestar Psicológico en un grupo de inmigrantes sudamericanos en Chile. Salud \& Sociedad, 1(1), 51-70.

Zapata, M., A. (2009). Familia transnacional y remesas: padres y madres migrantes. Revista Latinoamericana de Ciencias Sociales, Niñez y Juventud, 7(2), 1749-1769. 